\title{
SIMPLIFIED ANALYTICAL SOLUTION OF THE CONTACT PROBLEM ON INDENTATION OF A COATED HALF-SPACE BY A SPHERICAL PUNCH
}

\author{
EVGENIY V. SADYRIN ${ }^{1,2}$, ANDREY S. VASILIEV ${ }^{1,2}$, SERGEI S. VOLKOV ${ }^{1,2}$, \\ BORIS I. MITRIN ${ }^{1} \&$ SERGEI M. AIZIKOVICH ${ }^{1}$ \\ ${ }^{1}$ Research and Education Center "Materials", Don State Technical University, Russia \\ ${ }^{2}$ Research Institute for Mechanics, National Research Lobachevsky State University of Nizhni Novgorod, Russia
}

\begin{abstract}
This paper is devoted to construction of a mathematical model combining simplicity for practical usage and high accuracy. It is based on the solution of an axisymmetric contact problem on penetration of a rigid indenter into an elastic half-space with a functionally graded or homogeneous coating. The problem is reduced to solution of a dual integral equation. Asymptotically exact expressions for indentation force, depth, contact stiffness and distribution of contact pressures are obtained in simplified analytical form using one-parameter approximation of the integral equation kernel transform. Numerical calculations are provided for a number of homogeneous and functionally graded coatings. Accuracy of the solution is analyzed against ratio of Young's moduli of coating and substrate and the value of relative coating thickness.

Keywords: contact, penetration, spherical indenter, simple analytical solution, functionally graded coating.
\end{abstract}

\section{INTRODUCTION}

One of the ways to improve reliability and increase the service life of machine parts, elements of building structures, components of micro- and nanoelectronics as well as other products of modern industry is the modification of the initial surface of products by applying protective coatings. Such coatings can have a multilayer or functionally graded structure. In practice, nanoindentation is one of commonly used approaches to determine the mechanical characteristics of coatings.

To interpret the nanoindentation results, methods based on mathematical models that use solutions of classical contact problems for isotropic homogeneous materials are widely used. Oliver and Pharr proposed [1] and then modernized [2] a method for determining the hardness and Young's modulus of homogeneous isotropic materials, which is based on the Sneddon's solution for an axisymmetric indenter [3]. Field and Swain [4] for the interpretation of the spherical indentation results proposed a method that uses Hertz's solution of the problem of a spherical indenter penetration into a homogeneous half-space. Despite the fact that these methods do not take into account the influence of the substrate on the experimental results, under certain experimental conditions (indentation depth does not exceed $10 \%$ of the coating thickness [5]) and certain ratios of the mechanical properties of the coating and substrate [6][9] the properties of homogeneous coatings can be recovered. However, applying such methods in case of significant difference in Young's moduli of the coating and substrate can lead to significant underestimation or overestimation of reconstructed value of Young's modulus of the coating. In addition, this approach cannot be used to determine the Young's modulus of thin coatings, since the required indentation depth becomes comparable to the surface roughness height. In this situation, mathematical models based on contact problems for coated solids can be effectively used.

Plane and axisymmetric contact problems for elastic solids with coatings with arbitrarily varying elastic properties in depth were studied by Ke and Wang [10] and Liu et al. [11]. 
They used an approximation of the function describing the variation of the Young's modulus by a piecewise linear function. The singular integral equation of the problem was solved by the collocation method. Guler and Erdogan [12] constructed a solution of the plane contact problem on indentation considering the exponential variation of the elastic moduli by depth using similar collocation technique. Argatov et al. [13]-[16] developed mathematical models for nanoindentation of samples with a single-layer uniform thick coating using the indenters of various shapes.

In the papers by Aizikovich [21], Vasiliev et al. [17]-[19] and Volkov et al. [20] solution of axisymmetric and plane contact problems for elastic solids with a functionally graded coating were constructed using the bilateral asymptotic method. This approach has a few advantages. It effectively works for the entire range of values of the relative thickness of the coating; arbitrary variation of elastic properties in depth may be modeled. Also, the problem solutions are constructed in analytical form. However, the developed models are multiparameter, and it is rather difficult to use them in practice.

This paper is devoted to the construction of a simplified mathematical model of a spherical stamp indentation, allowing to take into account both the elastic properties of the coating and the contribution of the substrate. The model is based on the solution of an axisymmetric contact problem on indentation of an elastic half-space with functionally graded or homogeneous coating by a rigid punch. The solution is also constructed using the bilateral asymptotic method [21]. The main difference from the results obtained before is in the fact that the kernel transform is approximated by a ratio of two quadratic functions containing only one parameter. In this case, the scheme for construction of an approximated analytical solution is substantially simplified in comparison with the general case [17]-[20], in which the product of quadratic-fractional functions is used. The use of simple one-parameter approximation makes it possible to obtain the solution of the problem in an explicit analytical form, convenient in practice for engineering calculations.

\section{PROBLEM STATEMENT}

The mathematical model of the indentation of a coating-substrate system by a spherical indenter is based on the contact problem of the linear elasticity. The indenter is modeled by a rigid spherical punch of radius $R$ (the results can be easily generalized to the case of a deformable elastic indenter [22]). The sample is modeled by an elastic half-space consisting of the functionally graded or homogeneous elastic layer (coating) of thickness $H$ and a homogeneous half-space (substrate).

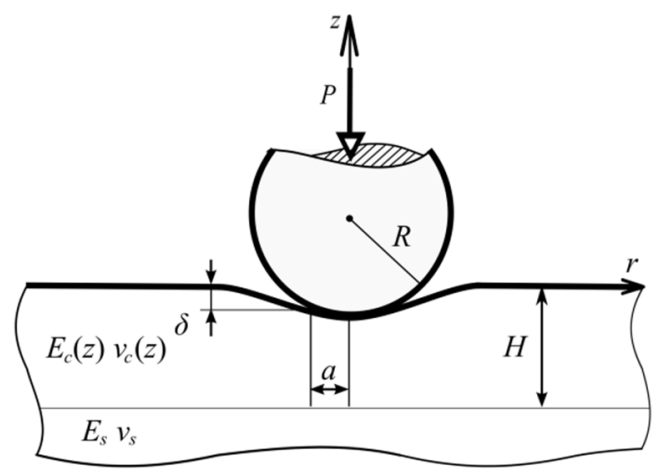

Figure 1: Statement of the contact problem. 
Let us consider the cylindrical coordinate system $(r, \varphi, z)$, the $z$ axis is orthogonal to the surface of the half-space and passes through the center of the punch. Under the action of the centrally applied force $P$, the punch is displaced in the negative direction of the $z$ axis by an amount $\delta$. We consider the frictional forces between the base of the punch and the elastic layer to be negligibly small. The Young's modulus and Poisson's ratio of the half-space vary by depth according to the following laws:

$$
\{E, v\}=\left\{\begin{array}{l}
\left\{E_{c}(z), v_{c}(z)\right\},-H \leq z \leq 0 ; \\
\left\{E_{s}, v_{s}=\text { const }\right\},-\infty<z<-H .
\end{array}\right.
$$

Here and after, index $(c)$ corresponds to the coating while index $(s)$ to the substrate.

Let the coordinate $r$ be related to the radius of the contact zone $a$. In this case the boundary conditions on the surface of the half-space have the following form

$$
z=0: \quad \tau_{z r}=0,\left\{\begin{array}{c}
\sigma_{z}=0, \quad r>1 \\
w=-\delta+a^{2} r^{2} / 2 R, r \leq 1 .
\end{array}\right.
$$

To obtain simpler equations the spherical shape of a punch is approximated by a paraboloid. It can be done with high accuracy if $R>>a$.

Complete adhesion at the interface between the coating and the substrate is assumed:

$$
z=-H: \quad \tau_{z r}^{(c)}=\tau_{z r}^{(s)}, \quad \sigma_{z}^{(c)}=\sigma_{z}^{(s)}, w^{(c)}=w^{(s)}, u^{(c)}=u^{(s)} .
$$

Here $u$ and $w$ are the displacements along $r$ and $z$ axes, respectively; $\sigma_{z}, \sigma_{\phi}, \sigma_{r}, \tau_{z r}$ are the components of the stress tensor. It is required to find the distribution of contact normal pressure:

$$
\left.\sigma_{z}\right|_{z=0}=-p(r), \quad r \leq 1 .
$$

We assume that both the stresses and displacements vanish at $r \rightarrow \infty$ and $z \rightarrow-\infty$. To determine the size of the contact area, we will use the classical assumption of zero contact stresses at the contact boundary: $p(1)=0$.

\section{PROBLEM SOLUTION}

Using Hankel integral transforms [23], the integral equation is obtained [17]:

$$
\int_{0}^{1} p(\rho) \rho d \rho \int_{0}^{\infty} L(u) \mathrm{J}_{0}\left(u r \lambda^{-1}\right) \mathrm{J}_{0}\left(u \rho \lambda^{-1}\right) d u=\lambda \Theta\left(\delta-a^{2} r^{2} / 2 R\right) a^{-1}, \quad r \leq 1 .
$$

Following notations were used above: $\lambda=H / a$ is the relative thickness of the coating; $L(u)$ is the kernel transform of the integral equation which is generally determined numerically from the Cauchy problem solution for a system of ordinary differential equations with variable coefficients [17]. Let $\Theta$ and $\Theta_{s}$ be constants characterizing the effective elastic properties of the surfaces of the coating and the substrate:

$$
\Theta=E_{c}(0) /\left(2\left(1-v_{c}^{2}(0)\right)\right), \quad \Theta_{s}=E_{s} /\left(2\left(1-v_{s}^{2}\right)\right) .
$$

To solve eqn (5) we approximate the function $L(u)$ by the expression 


$$
L(u) \approx L_{N}(u)=\prod_{i=1}^{N}\left(u^{2}+A_{i}^{2}\right) /\left(u^{2}+B_{i}^{2}\right), A_{i}, B_{i} \in C .
$$

Then the solution of the problem has the form analogous to [24]

$$
p(r)=\frac{4 a \Theta_{s}}{\pi R}\left[\sqrt{1-r^{2}}+\sum_{i=1}^{N} C_{i} \frac{A_{i}}{\lambda} \int_{r}^{1} \frac{\sinh \left(A_{i} \lambda^{-1} t\right)}{\sqrt{t^{2}-r^{2}}} d t\right], \quad 0 \leq r \leq 1 .
$$

Constants $C_{i}(i=1, \ldots, N)$ are determined from a system of linear algebraic equations:

$$
\sum_{i=1}^{N} C_{i} A_{i}\left[\frac{B_{k} \sinh \left(A_{i} \lambda^{-1}\right)+A_{i} \cosh \left(A_{i} \lambda^{-1}\right)}{B_{k}^{2}-A_{i}^{2}}\right]=-\frac{\lambda\left(B_{k}+\lambda\right)}{B_{k}^{2}}, \quad k=1,2, \ldots, N .
$$

To analyze the experiments on nanoindentation in practice, the indentation stiffness function is used $S=d P / d \delta$ [1]. Its value can be determined experimentally from the slope angle of tangent line to the unloading curve (of the graph demonstrating the dependence of indenter displacement from the indentation force) at the upper point of this curve (at the maximum depth of the indenter displacement).

Expressions for punch displacement, indentation force and stiffness have the form [24]:

$$
\begin{gathered}
\delta=\frac{a^{2}}{R}\left[1+2 \sum_{i=1}^{N} C_{i} \cosh \left(\frac{A_{i}}{\lambda}\right)+2 \lambda^{2} \sum_{i=1}^{N}\left(\frac{1}{A_{i}^{2}}-\frac{1}{B_{i}^{2}}\right)\right], \\
P=\frac{8 a^{3} \Theta_{s}}{3 R}\left[1+3 \sum_{i=1}^{N} C_{i}\left(\cosh \left(\frac{A_{i}}{\lambda}\right)-\frac{\lambda}{A_{i}} \sinh \left(\frac{A_{i}}{\lambda}\right)\right)\right], \\
S=\frac{d P}{d \delta}=4 a \Theta_{s} \frac{1+3 \sum_{i=1}^{N} C_{i}\left(\cosh \left(A_{i} \lambda^{-1}\right)-A_{i}^{-1} \lambda \sinh \left(A_{i} \lambda^{-1}\right)\right)}{1+2 \sum_{i=1}^{N} C_{i} \cosh \left(A_{i} \lambda^{-1}\right)+2 \lambda^{2} \sum_{i=1}^{N}\left(A_{i}^{-2}-B_{i}^{-2}\right)} .
\end{gathered}
$$

It is proved [24] that the obtained solution (8)-(12) is asymptotically exact for large and small values of the geometrical parameter of the problem $\lambda$. The error of the solution for intermediate values is related to the accuracy of the approximation of the kernel transform. On example of the torsion problem it was shown that these errors are of the same order of magnitude [25]. Then

In order to simplify the solution as much as possible, let us assume that $N=1$ in eqn (7).

$$
L(u) \approx\left(u^{2}+A^{2}\right) /\left(u^{2}+B^{2}\right) .
$$

It is important to note that the approximation of the kernel transform is constructed in such a way that $L_{N}(0)=L(0)$ is satisfied. We introduce a parameter $\beta=\Theta_{s} / \Theta$ characterizing the "softness" of the coating. Coatings with $\beta>1$ we call "soft", while with $\beta<1$ we will call "hard" coatings. We also take into account that $L(0)=\beta^{-1}$ [24], hence $B=\sqrt{\beta} A$. Thus, eqns (8)-(12) may be simplified 


$$
\begin{gathered}
p(r)=\frac{4 a \Theta_{s}}{\pi R}\left[\sqrt{1-r^{2}}+\frac{\left(\sqrt{\beta}+\lambda A^{-1}\right)\left(\beta^{-1}-1\right)}{\left(\sqrt{\beta} \sinh \left(A \lambda^{-1}\right)+\cosh \left(A \lambda^{-1}\right)\right)} \int_{r}^{1} \frac{\sinh \left(A \lambda^{-1} t\right)}{\sqrt{t^{2}-r^{2}}} d t\right], \quad 0 \leq r \leq 1, \\
\delta=a^{2}\left(1+2 \sqrt{\beta} \Delta_{0}\right) / R, \\
P=8 a^{3} \Theta_{s}\left(1+3\left(\sqrt{\beta}+\lambda A^{-1}\right) \Delta_{0}\right) /(3 R), \\
S=4 a \Theta_{s}\left(1+\frac{\sqrt{\beta}+3 \lambda A^{-1}}{1+2 \sqrt{\beta} \Delta_{0}} \Delta_{0}\right), \\
\Delta_{0}=\frac{\lambda}{A^{2}}\left(\beta^{-1}-1\right)\left(\frac{A-\lambda \tanh \left(A \lambda^{-1}\right)}{1+\sqrt{\beta} \tanh \left(A \lambda^{-1}\right)}\right) .
\end{gathered}
$$

\section{NUMERICAL RESULTS}

To illustrate the simplified solutions obtained, we consider a set of homogeneous and functionally graded coatings. Let the Young's modulus of the substrate be $E_{0}=$ const, and the Poisson's ratio of both the coating and the substrate is 0.33 . Let us consider a number of homogeneous coatings for which $\beta=0.1,0.2,0.5,2,5,10$ (we will call them coatings $1-6$, respectively) and four functionally graded coatings:

$$
\text { coatings } 7 \text { and 8: } E_{c}^{\text {lin }}=E_{0}\left(\beta-(1-\beta) z H^{-1}\right), \beta=0.5,2 \text {. }
$$

coatings 9 and 10: $E_{c}^{\exp }=E_{0}\left(\beta e^{k}-1-(\beta-1) e^{-k z H^{-1}}\right)\left(e^{k}-1\right)^{-1}, k=-3 ; \beta=0.5,2$.

Graphs of kernel transforms of integral equations for coatings 1-10 are shown in Fig. 2. Kernel transforms for homogeneous coatings converge much faster to unity as $u \rightarrow \infty$. Kernel transforms for soft coatings 4-6, 8, 10 converge more slowly to the value of $\beta^{-1}$ as $u \rightarrow 0$ than the kernel transforms for hard coatings $1-3,7,9$. The smaller value of $\beta$, the slower convergence is.

Table 1 shows the values of error of kernel transforms approximation for coatings 1-10 using multiparameter approximation (7) and one-parameter approximation (13). As can be seen, the more the value of $\beta$ differs from unity (upward or downward), the higher the error of one-parameter approximation. However, for many of the examples considered, even a oneparameter approximation allows to obtain a sufficiently high accuracy. The error of multiparameter approximation for all the examined coatings is not exceeding $0.20 \%$, that indicates the high accuracy of the results.

To analyze the distribution of contact stresses, it is convenient to introduce dimensionless quantities. Let us denote $p_{\text {hom }}(r), P_{\text {hom }}, \delta_{\text {hom }}, S_{\text {hom }}$ as the contact stresses, indentation force, punch displacement and indentation stiffness, respectively, in the Hertzian contact (for a homogeneous half-space with elastic properties that coincide with the substrate). We introduce dimensionless variables and functions:

$$
p_{0}(r)=\frac{p(r)}{p_{\text {hom }}(0)}=\frac{\pi R p(r)}{4 a \Theta_{s}}, \delta_{0}=\frac{\delta}{\delta_{\text {hom }}}=\frac{\delta R}{a^{2}}, P_{0}=\frac{P}{P_{\text {hom }}}=\frac{3 R P}{8 a^{3} \Theta_{s}}, S_{0}=\frac{S}{S_{\text {hom }}}=\frac{S}{4 a \Theta_{s}} \text {. }
$$

In order to analyze the difference in the distribution of contact stresses for a half-space with and without coating, we also consider the relative contact stresses:

$$
p_{\text {rel }}(r)=p(r) / p_{\text {hom }}(r) \text {. }
$$




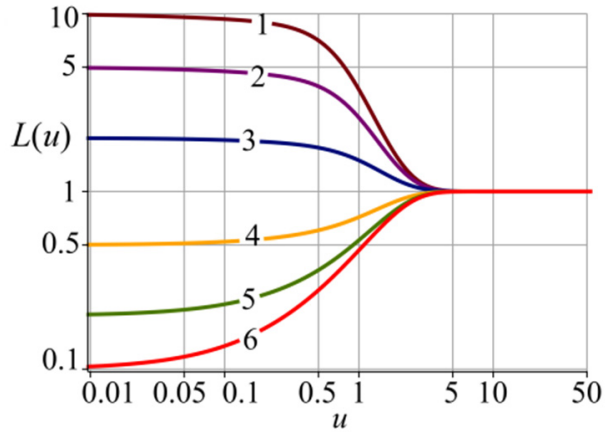

(a)

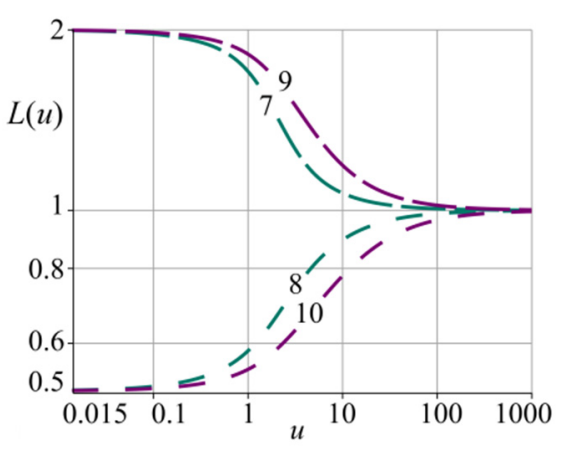

(b)

Figure 2: Kernel transforms for (a) homogeneous; and (b) functionally graded coatings.

Table 1: Values of error of kernel transforms approximation for coatings 1-10.

\begin{tabular}{|c|l|c|c|c|}
\hline \multirow{2}{*}{$\begin{array}{c}\text { Coating } \\
\text { no. }\end{array}$} & Type of inhomogeneity & \multirow{2}{*}{$\beta$} & \multicolumn{2}{|c|}{ Approximation error } \\
\cline { 3 - 5 } & & $N=1$ & $N>1$ \\
\hline 1 & Homogeneous & 0.1 & $18.61 \%$ & $0.15 \%$ \\
\hline 2 & Homogeneous & 0.2 & $11.78 \%$ & $0.10 \%$ \\
\hline 3 & Homogeneous & 0.5 & $3.80 \%$ & $0.08 \%$ \\
\hline 4 & Homogeneous & 2 & $4.30 \%$ & $0.05 \%$ \\
\hline 5 & Homogeneous & 5 & $12.6 \%$ & $0.11 \%$ \\
\hline 6 & Homogeneous & 10 & $20.70 \%$ & $0.20 \%$ \\
\hline 7 & Linear & 0.5 & $3.80 \%$ & $0.05 \%$ \\
\hline 8 & Linear & 2 & $6.80 \%$ & $0.06 \%$ \\
\hline 9 & Exponential & 0.5 & $6.84 \%$ & $0.08 \%$ \\
\hline 10 & Exponential & 2 & $9.34 \%$ & $0.07 \%$ \\
\hline
\end{tabular}

Figs 3-5 demonstrate the graphs of indentation force, depth and stiffness for homogeneous coatings $1-6$ and functionally graded coatings $7-10$ for $N=1$ and $N>>1$. Logarithmic scale is used. For $\lambda \rightarrow 0$ the values of $P_{0}, \delta_{0}, S_{0}$ tend to unit, which means that the force, depth and stiffness almost equal to the values corresponding to the Hertzian contact.

For $\lambda \rightarrow \infty$, values of $P_{0}$ and $S_{0}$ tend to $\beta^{-1}$, and $\delta_{0}$ to 1 . This means that these values coincide with the Hertzian ones for the elasticity moduli of the coating surface $E_{c}(0), v_{c}(0)$. The behavior of $P_{0}$ and $S_{0}$ in a region of intermediate values of $\lambda$ is very similar to the graph of the kernel transform (see Fig. 2). The graphs of $P_{0}$ and $S_{0}$ constructed using one-parameter and multiparameter approximations are sufficiently close for all the coatings considered, the graphs of $\delta_{0}$ are less close to each other.

Figs 6 and 7 illustrate the distribution of contact pressure and relative contact pressure for coatings 4 and 10. One-parameter approximation allows to achieve a fairly good coincidence of the contact pressure distributions for small and large $\lambda$ values for all the coatings considered, as well as for the intermediate $\lambda$ in the case of a small ratio of the coating and substrate elastic moduli. 


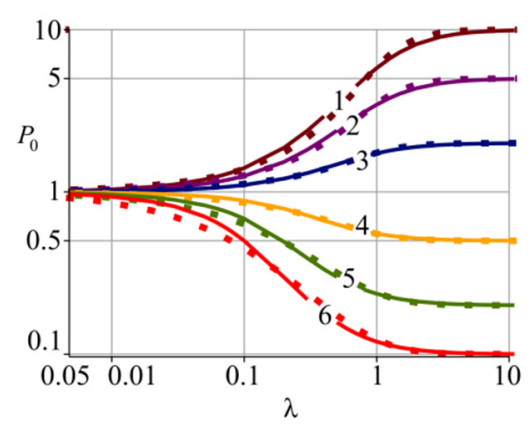

(a)

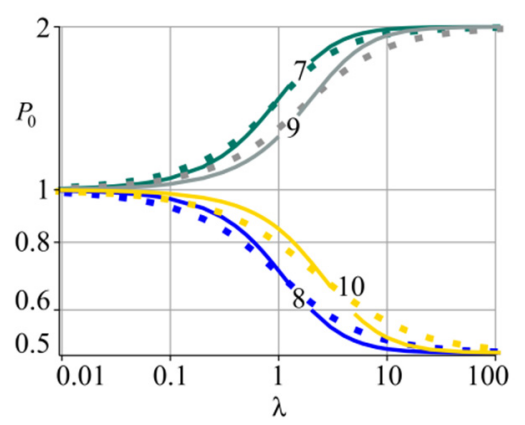

(b)

Figure 3: Indentation force for (a) homogeneous; and (b) functionally graded coatings for $N=1$ (solid line) and $N>>1$ (dotted line).

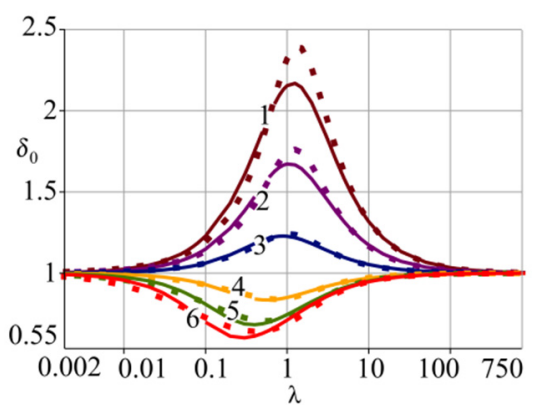

(a)

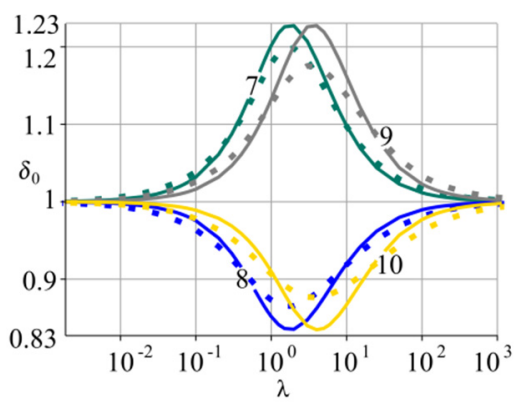

(b)

Figure 4: Indentation depth for (a) homogeneous; and (b) functionally graded coatings for $N=1$ (solid line) and $N>>1$ (dotted line).

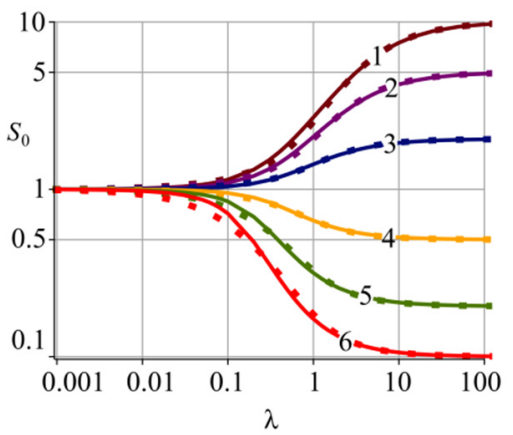

(a)

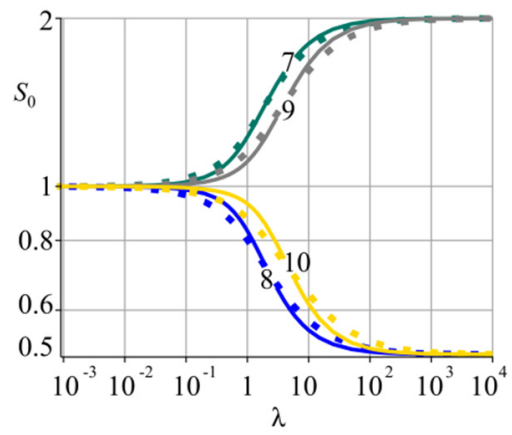

(b)

Figure 5: Indentation stiffness for (a) homogeneous; and (b) functionally graded coatings for $N=1$ (solid line) and $N>>1$ (dotted line). 


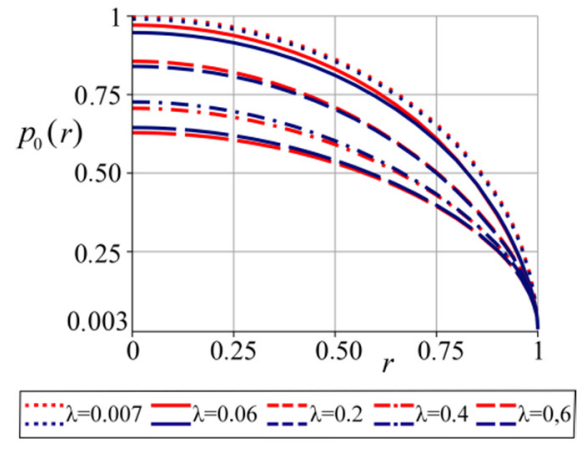

(a)

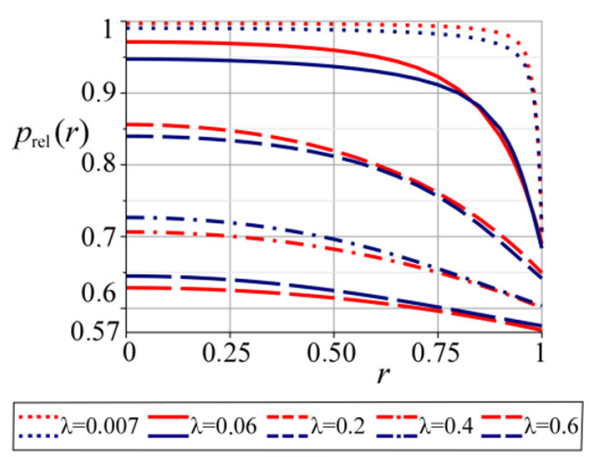

(b)

Figure 6: Distribution of (a) dimensionless contact stresses; and (b) relative contact stresses for a homogeneous coating $4(\beta=2)$ for $N=1$ (red lines) and $N \gg>1$ (blue lines) for various $\lambda$.

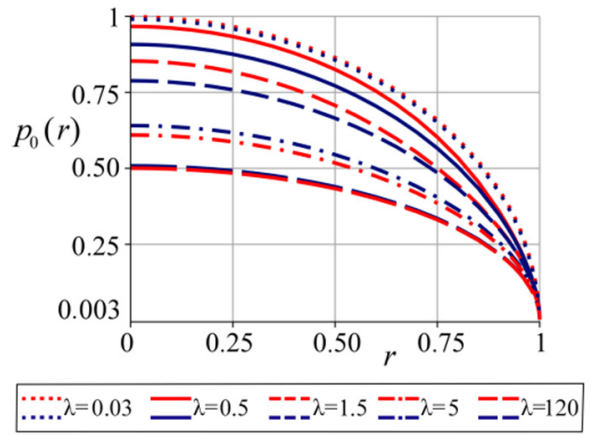

(a)

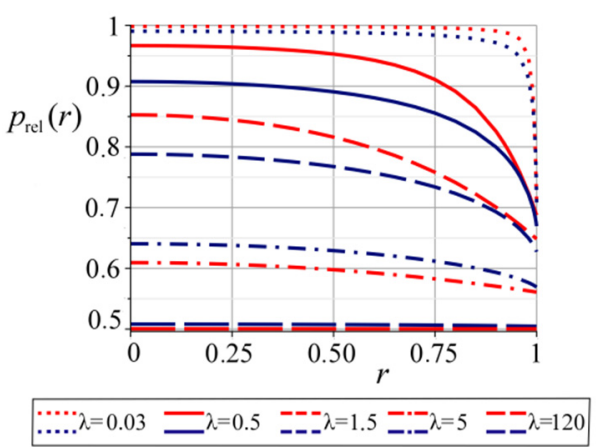

(b)

Figure 7: Distribution of dimensionless contact stresses (a) and relative contact stresses (b) for a homogeneous coating $10(\beta=2)$ for $N=1$ (red lines) and $N>>1$ (blue lines) for various $\lambda$.

For a more detailed analysis of the accuracy of simplified solutions based on oneparameter approximation, we compare them with solutions obtained with multiparameter (much more accurate) approximation. Relative error of the simplified solution was calculated as:

$$
\Delta_{p}=\left|1-\frac{p_{N=1}(r)}{p_{N>1}(r)}\right| \cdot 100 \% .
$$

Fig. 8 demonstrates three-dimensional graphs of the relative error $\Delta_{p}(r)$ for coatings 7 and 1. For these coatings the one-parameter approximation of the kernel transform has the minimal value of $3.5 \%$ (for coatings 7 and 3 ) and maximal value of $29.30 \%$ (for coating 1 ), see Table 1. As can be seen, the maximum error in contact pressures is comparable to the error of approximation of the transform (3.80\% for coating 7 and $18.61 \%$ for coating 1$)$. In 


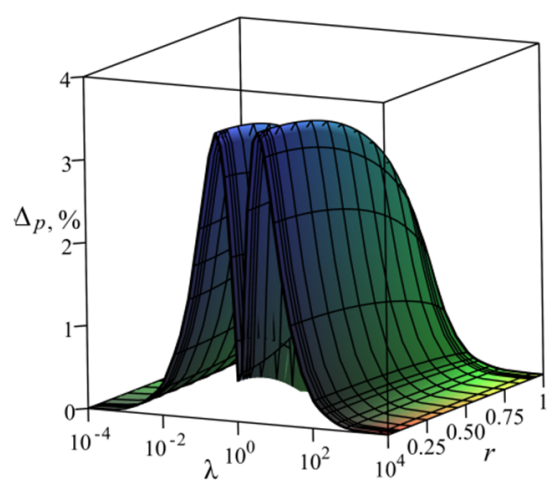

(a)

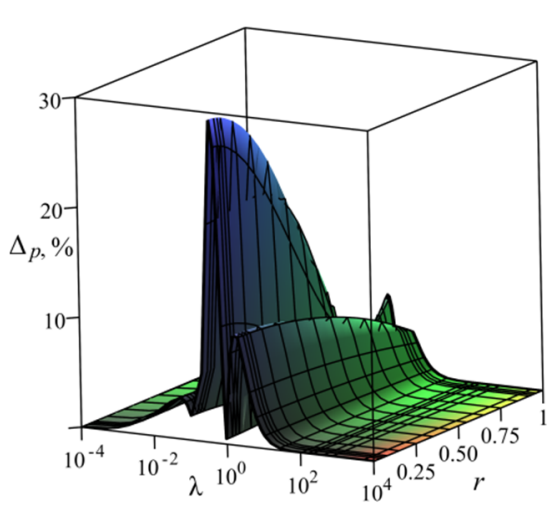

(b)

Figure 8: Relative error of the simplified solution for (a) coating 7; and (b) coating 1.

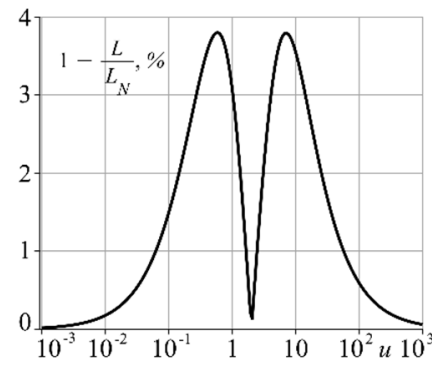

(a)

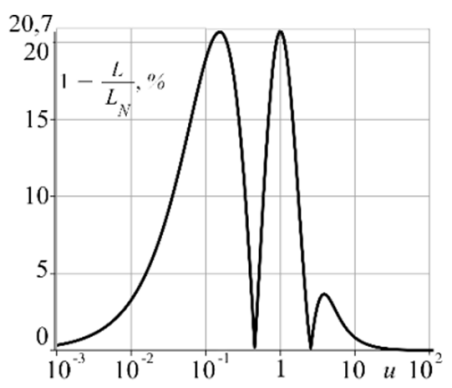

(b)

Figure 9: Relative error of simplified approximation of the kernel transform for (a) coating 3 ; and (b) coating 6.

the same way as the error graph of a one-parameter kernel transform approximation has two distinct hillocks (see Fig. 9), the error of contact pressures have them for any fixed value of $0 \leq r<1$, with the exception of a small neighborhood of $r \approx 1$.

It can be seen from Fig. 8(b) that even with a relatively low accuracy of approximation (coating 1) for sufficiently large and small values of $\lambda$, the error of the solution is small. Let $\lambda_{0}$ be the value at which the maximum relative error of contact stresses is less than $1 \%$ for all $\lambda<\lambda_{0}$. Similarly, $\lambda_{\infty}$ is such a value that for all $\lambda>\lambda_{\infty}$ the error is less than $1 \%$. Table 2 lists the values of $\lambda_{0}$ and $\lambda_{\infty}$ for all the 10 coatings. It is important to note that the maximum value of $\Delta_{p}(r)$ is calculated for $0 \leq r<r_{0}$, where $r_{0}$ is a certain value close to unit. In the vicinity of $r \approx 1$, for small values of $\lambda$, the relative error of contact pressures may be higher than $1 \%$. This is due to the peculiarity of the behavior of the relative contact pressure distribution in this region. As was noted earlier [18], for any small value of $\lambda$ there is a small neighborhood of $r \approx 1$ where $p_{\text {rel }} \approx \beta^{-1 / 2}$. Since both the one-parameter and multiparameter approximations exactly coincide with the transform values for $u=0$ and $u \rightarrow \infty$, then $\Delta_{p}(r) \rightarrow 0$ is fulfilled for $r \rightarrow 1$. However, a narrow area is observed to the left of $r=1$ where the value of $\Delta_{p}(r)$ is quite high even for very small values of $\lambda$. It should also be noted that the absolute values of 
Table 2: Values of $\lambda_{0}$ and $\lambda_{\infty}$ for coatings $1-10$.

\begin{tabular}{|c|c|l|c|c|c|}
\hline $\begin{array}{c}\text { Coating } \\
\text { no. }\end{array}$ & $\beta$ & Type of inhomogeneity & $\lambda_{0}$ & $\lambda_{\infty}$ & $\max \left(\Delta_{p}\right)$ \\
\hline 1 & 0.1 & Homogeneous & 0.003 & 15 & $29.30 \%$ \\
\hline 2 & 0.2 & Homogeneous & 0.005 & 10 & $14.52 \%$ \\
\hline 3 & 0.5 & Homogeneous & 0.03 & 50 & $3.51 \%$ \\
\hline 4 & 2 & Homogeneous & 0.01 & 4 & $4.22 \%$ \\
\hline 5 & 5 & Homogeneous & 0.002 & 5 & $12.36 \%$ \\
\hline 6 & 10 & Homogeneous & 0.001 & 6 & $23.14 \%$ \\
\hline 7 & 0.5 & Linear & 0.03 & 50 & $3.50 \%$ \\
\hline 8 & 2 & Linear & 0.02 & 100 & $6.51 \%$ \\
\hline 9 & 0.5 & Exponential & 0.03 & 120 & $6.33 \%$ \\
\hline 10 & 2 & Exponential & 0.03 & 300 & $9.13 \%$ \\
\hline
\end{tabular}

Table 3: Approximation error for $P, S$ and $\delta$ for coatings 1-10.

\begin{tabular}{|c|c|l|c|c|c|}
\hline $\begin{array}{c}\text { Coating } \\
\text { no. }\end{array}$ & $\beta$ & Type of inhomogeneity & $\Delta_{P}$ & $\Delta_{S}$ & $\Delta_{\delta}$ \\
\hline 1 & 0.1 & Homogeneous & $10.95 \%$ & $7.33 \%$ & $10.14 \%$ \\
\hline 2 & 0.2 & Homogeneous & $5.94 \%$ & $3.65 \%$ & $5.73 \%$ \\
\hline 3 & 0.5 & Homogeneous & $2.93 \%$ & $2.71 \%$ & $1.35 \%$ \\
\hline 4 & 2 & Homogeneous & $3.02 \%$ & $2.78 \%$ & $1.90 \%$ \\
\hline 5 & 5 & Homogeneous & $9.65 \%$ & $9.11 \%$ & $5.25 \%$ \\
\hline 6 & 10 & Homogeneous & $17.53 \%$ & $16.57 \%$ & $8.35 \%$ \\
\hline 7 & 0.5 & Linear & $3.01 \%$ & $2.48 \%$ & $2.44 \%$ \\
\hline 8 & 2 & Linear & $4.91 \%$ & $4.64 \%$ & $3.50 \%$ \\
\hline 9 & 0.5 & Exponential & $5.36 \%$ & $4.03 \%$ & $4.53 \%$ \\
\hline 10 & 2 & Exponential & $6.62 \%$ & $6.28 \%$ & $4.77 \%$ \\
\hline
\end{tabular}

the contact pressure are small near $r=1$. That is why the value of relative error in this region is less important.

Similar to the contact stresses, let us analyze error of simplified expressions for the indentation force, depth and stiffness using following expressions:

$$
\Delta_{\{P, S, \delta\}}=\left|1-\frac{\{P, S, \delta\}_{N=1}}{\{P, S, \delta\}_{N>1}}\right| \cdot 100 \% .
$$

Approximation error for $P, S$ and $\delta$ are presented in the Table 3. For all considered coatings the values are even less than the error of approximation of the kernel transform. Graphs of relative errors of $P, S$ and $\delta$ are illustrated for the coating 3 in Fig. 10. 

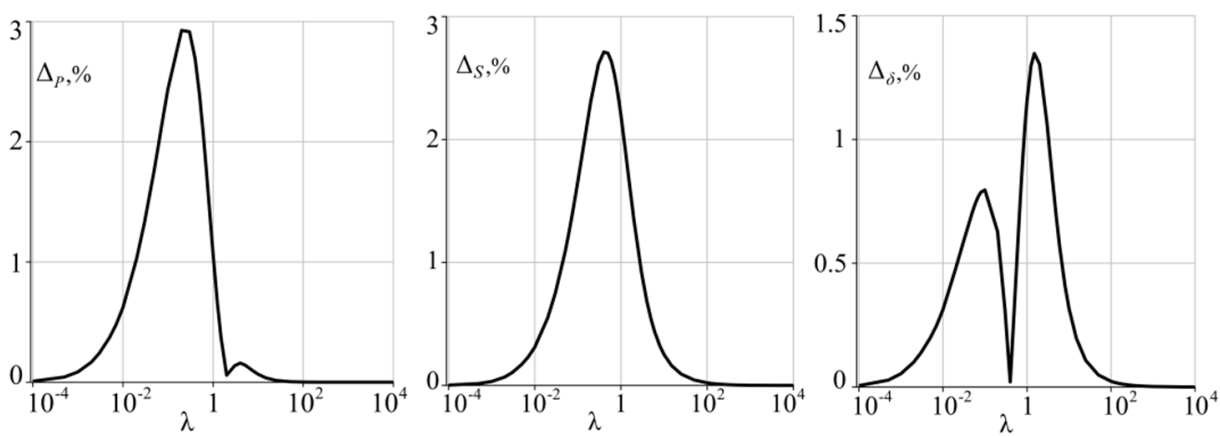

Figure 10: Relative error of the simplified solution for coating 3.

\section{CONCLUSION}

Simplified analytical expressions for the contact pressure, indentation force, depth and stiffness are obtained for the problem on indentation of a half-space with a homogeneous or functionally graded coating by a spherical punch. The accuracy of the results is studied in details. It is shown that the simplified formulas have sufficiently high accuracy for thin and thick coatings and for coatings of arbitrary thickness in the case of a relatively small difference in the elastic moduli of the coating and the substrate. For these cases, the obtained formulas can be used to recover coating characteristics from the indentation experiment. The approach can be applied to wide range of problems which can be reduced to a system of dual integral equations, including thermoelastic [26], electroelastic [27], fracture [28] problems.

\section{ACKNOWLEDGEMENTS}

Authors acknowledge the support of the Ministry of Education and Science of Russia in the framework of Governmental Assignment no. 9.1481.2017/4.6. E.V. Sadyrin was supported by the scholarship of the President of Russia no. SP-3672.2018.1.

\section{REFERENCES}

[1] Oliver, W.C. \& Pharr, G.M., An improved technique for determining hardness and elastic modulus using load and displacement sensing indentation experiments. Journal of Materials Research, 7(6), pp. 1564-1583, 1992. DOI: 10.1557/jmr.1992.1564.

[2] Oliver, W.C. \& Pharr, G.M., Measurement of hardness and elastic modulus by instrumented indentation: Advances in understanding and refinements to methodology. Journal of Materials Research, 19(1), pp. 3-20, 2004.

DOI: 10.1557/jmr.2004.0002.

[3] Sneddon, I.N., The relation between load and penetration in the axisymmetric Boussinesq problem for a punch of arbitrary profile. International Journal of Engineering Science, 3(1), pp. 47-57, 1965. DOI: 10.1016/0020-7225(65)90019-4.

[4] Field, J.S. \& Swain, M.V., Determining the mechanical properties of small volumes of material from submicrometer spherical indentations. Journal of Materials Research, 10(1), pp. 101-112, 1995. DOI: 10.1557/jmr.1995.0101.

[5] Bückle, H., The Science of Hardness Testing and its Research Applications, eds. J.H. Westbrook \& H. Conrad, American Society For Metals: Ohio, pp. 453-494, 1973. 
[6] Chen, J. \& Bull, S.J., On the factors affecting the critical indenter penetration for measurement of coating hardness. Vacuum, 83(6), pp. 911-920, 2009.

DOI: $10.1016 /$ j.vacuum.2008.11.007.

[7] Cai, X. \& Bangert, H., Hardness measurements of thin films-determining the critical ratio of depth to thickness using FEM. Thin Solid Films, 264(1), pp. 59-71, 1995. DOI: 10.1016/0040-6090(95)06569-5.

[8] Veprek-Heijman, M.G.J. \& Veprek, S., The deformation of the substrate during indentation into superhard coatings: Bückle's rule revised. Surface and Coatings Technology, 284, pp. 206-214, 2015. DOI: 10.1016/j.surfcoat.2015.10.064.

[9] Panich, N. \& Sun, Y., Effect of penetration depth on indentation response of soft coatings on hard substrates: a finite element analysis. Surface and Coatings Technology, 182(2-3) pp. 342-350, 2004. DOI: 10.1016/j.surfcoat.2003.07.002.

[10] Ke, L.L. \& Wang, Y.S., Two-dimensional contact mechanics of functionally graded materials with arbitrary spatial variations of material properties. International Journal of Solids and Structures, 43(18-19), pp. 5779-5798, 2006.

DOI: $10.1016 /$ j.ijsolstr.2005.06.081.

[11] Liu, T.J., Wang, Y.S. \& Zhang, C., Axisymmetric frictionless contact of functionally graded materials. Archive of Applied Mechanics, 78(4), pp. 267-282, 2008.

DOI: 10.1007/s00419-007-0160-y.

[12] Guler, M.A. \& Erdogan, F., Contact mechanics of graded coatings. International Journal of Solids and Structures, 41(14), pp. 3865-3889, 2004.

DOI: 10.1016/j.ijsolstr.2004.02.025.

[13] Argatov, I., Frictionless and adhesive nanoindentation: Asymptotic modeling of size effects. Mechanics of Materials, 42(8), pp. 807-815, 2010.

DOI: 10.1016/j.mechmat.2010.04.002.

[14] Argatov, I.I. \& Sabina, F.J., Spherical indentation of a transversely isotropic elastic half-space reinforced with a thin layer. International Journal of Engineering Science, 50(1), pp. 132-143, 2012. DOI: 10.1016/j.ijengsci.2011.08.009.

[15] Argatov, I.I. \& Sabina, F.J., Asymptotic analysis of the substrate effect for an arbitrary indenter. The Quarterly Journal of Mechanics and Applied Mathematics, 66(1), pp. 75-95, 2013. DOI: 10.1093/qjmam/hbs020.

[16] Argatov, I.I. \& Sabina, F.J., Small-scale indentation of an elastic coated half-space: the effect of compliant substrate. International Journal of Engineering Science, 104, pp. 87-96, 2016. DOI: 10.1016/j.ijengsci.2016.04.008.

[17] Vasiliev, A.S., Volkov, S.S. \& Aizikovich, S.M., Indentation of an axisymmetric punch into an elastic transversely-isotropic half-space with functionally graded transversely-isotropic coating. Materials Physics and Mechanics, 28, pp. 11-15, 2016.

[18] Vasiliev, A.S., Volkov, S.S., Belov, A.A., Litvinchuk, S.Y. \& Aizikovich, S.M., Indentation of a hard transversely isotropic functionally graded coating by a conical indenter. International Journal of Engineering Science, 112, pp. 63-75, 2017.

DOI: 10.1016/j.ijengsci.2016.12.002.

[19] Vasiliev, A., Volkov, S., Aizikovich, S. \& Jeng, Y.R., Axisymmetric contact problems of the theory of elasticity for inhomogeneous layers. ZAMM-Journal of Applied Mathematics and Mechanics/Zeitschrift für Angewandte Mathematik und Mechanik, 94(9), pp. 705-712, 2014. DOI: 10.1002/zamm.201300067.

[20] Volkov, S., Aizikovich, S., Wang, Y.S. \& Fedotov, I., Analytical solution of axisymmetric contact problem about indentation of a circular indenter into a soft functionally graded elastic layer. Acta Mechanica Sinica, 29(2), pp. 196-201, 2013. DOI: $10.1007 / \mathrm{s} 10409-013-0022-5$. 
[21] Aizikovich, S.M., Asymptotic solutions of contact problems of elasticity theory for media inhomogeneous in depth. Journal of Applied Mathematics and Mechanics, 46(1), pp. 116-124, 1982. DOI: 10.1016/0021-8928(82)90091-0.

[22] Kudish, I.I., Volkov, S.S., Vasiliev, A.S. \& Aizikovich, S.M., Lubricated point heavily loaded contacts of functionally graded materials. Part 1. Dry contacts. Mathematics and Mechanics of Solids, 23(7), pp. 1061-1080, 2017.

DOI: $10.1177 / 1081286517704689$.

[23] Uflyand, Ya.S., Integral Transformations In Problems of the Theory of Elasticity, Nauka: Leningrad, 1967.

[24] Volkov, S.S., Vasiliev, A.S., Aizikovich, S.M., Seleznev, N.M. \& Leontieva, A.V., Stress-strain state of an elastic soft functionally-graded coating subjected to indentation by a spherical punch. PNRPU Mechanics Bulletin, (4), pp. 20-34, 2016. DOI: 10.15593/perm.mech/2016.4.02.

[25] Aizikovich, S.M. \& Vasiliev, A.S., A bilateral asymptotic method of solving the integral equation of the contact problem of the torsion of an elastic half-space inhomogeneous in depth. Journal of Applied Mathematics and Mechanics, 77(1), pp. 91-97, 2013. DOI: 10.1016/j.jappmathmech.2013.04.011.

[26] Krenev, L.I., Aizikovich, S.M., Tokovyy, Y.V. \& Wang, Y.-C., Axisymmetric problem on the indentation of a hot circular punch into an arbitrarily nonhomogeneous half-space. International Journal of Solids and Structures, 59, pp. 18-28, 2015. DOI: 10.1016/j.ijsolstr.2014.12.017.

[27] Volkov, S.S., Vasiliev, A.S., Aizikovich, S.M. \& Mitrin, B.I., Axisymmetric indentation of an electroelastic piezoelectric half-space with functionally graded piezoelectric coating by a circular punch. Acta Mechanica, pp. 1-14, 2017. DOI: 10.1007/s00707-017-2026-x.

[28] Aizikovich, S.M., Galybin, A.N. \& Krenev, L.I., Semi-analytical solution for mode I penny-shaped crack in a soft inhomogeneous layer. International Journal of Solids and Structures, 53, pp. 129-137, 2015. DOI: 10.1016/j.ijsolstr.2014.10.010. 